\title{
IDADE DAS ÁGUAS SUBTERRÂNEAS NO AQUIFERO BEBERIBE NA REGIÃO COSTEIRA DE OLINDA-GOIANA, ESTADO DE PERNAMBUCO*
}

\author{
EMÍLIO CUSTÓDIO GIMENA**, WALDEMIR BARBOSA DE CRUZ***, \\ ADELBANI BRAZ DA SILVA***, FERNANDO GOMES JARDIM*** e \\ CARLOS ALBERTO DE MELLO PEIXOTO***
}

Recife - Pernambuco - Brasil

\begin{abstract}
Samples of ground water of the Beberibe aquifer in the seashore region Olinda-Goiana, State of Pernambuco, were analysed by tritium and radiocarbon contentes. The analyses indicated that they are ancient waters, aging till 20.000 years, specially in the lowest parts of the seashore zone, where they are practically under stationary conditions. On the transition zone between the upper and lower aquifer waters of considerably old ages are still encountered. This-also enhances the existence of an ascendent vertical water flow in these zones. In the recharge area the sampled water is about 1.750 years old, which indicates that the circulation is limited to the upper part of the aquifer, and the movement is much in deeper levels. There are no evidencès of contamination by direct infiltration of sea waters, although the occurence of little infiltration of superficial salty waters is possible.
\end{abstract}

INTRODUÇÃo Durante o levantamento dos recursos hídricos da região costeira dos Estados de Pernambuco, Paraíba è Rio Grande do Norte para fins de aproveitamento integrado, executado pela SUDENE, através da empresa Organização e Engenharia S.A., foram efetuadas algumas determinações do conteúdo de radiocarbono e trítio de águas subterrâneas do aquífero Beberibë, entre .Olinda e Goiana (Fig. 1).

As amostras analisadas foram coletadas em poços e furos piezométricos existentes ou especialmente perfurados para os estudos, que captam águas de vários níveis da parte inferior e superior do aquífero.

Os resultados obtidos permitiram determinar a idade das águas e fornecer informações sobre as condiçōes de fluxo subterrâneo e suas relações com as águas do mar.

* Estudo realizado pela SUDENE - DRN - CONESP, através da OESA - Organização e Engenharia S.A., como parte do programa "Estudos de Reconhecimento e Estudos Hidrogeológicos para Aproveitamento Integrado da Regiāo Centro-Oriental da Bacia Potiguar e da Bacia Costeira de Pernambuco - Paraíba".

* Consultor da OESA e Diretor do Curso Internacional de Hidlologia Subterranea, Beethoven 15 , 3․, Barcelona - Espanha.

** OESA … Organização e Engenharia S.A., Rio de Janciro Brasil. 


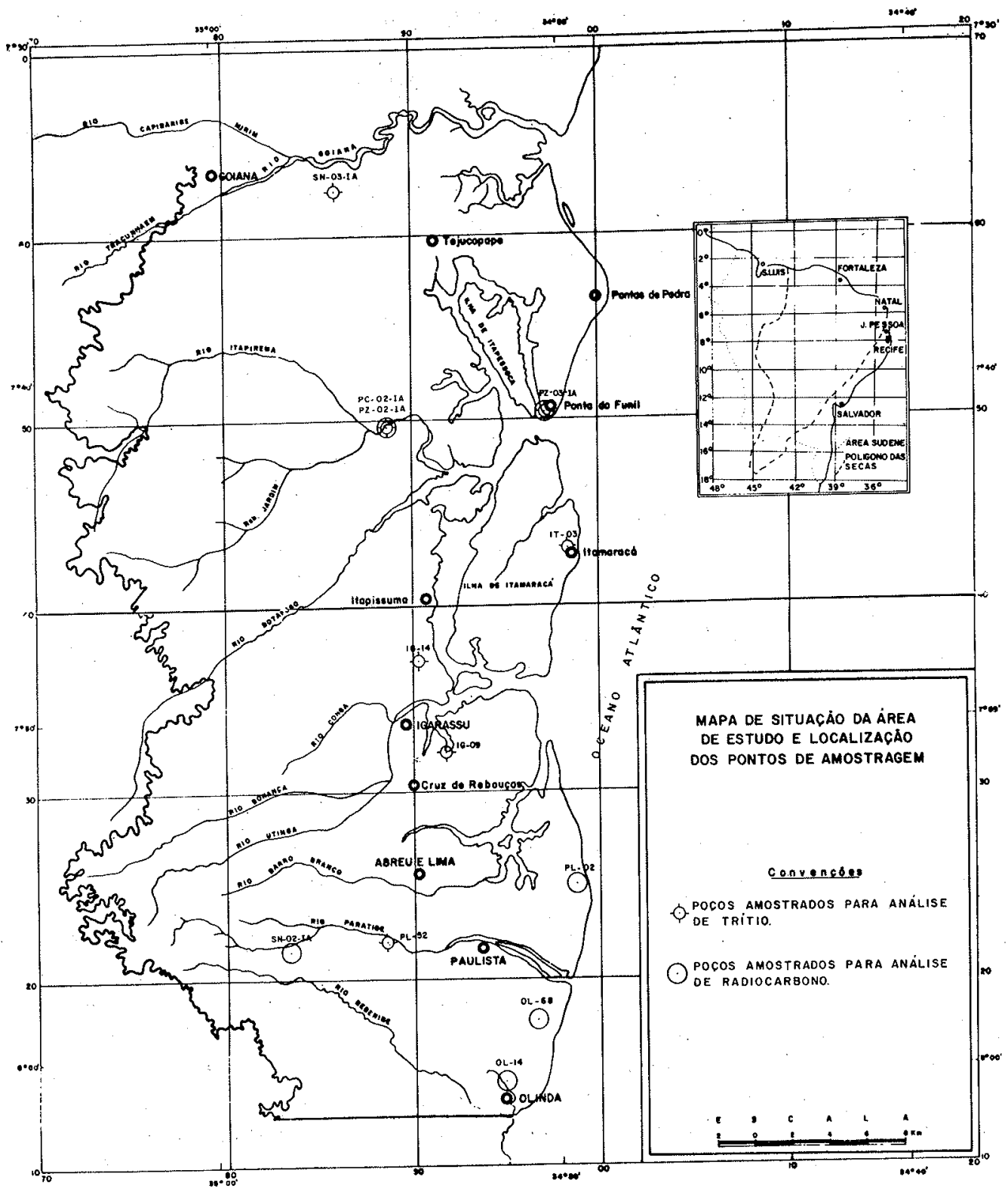

Figura 1

CONDIÇÕES HIDROGEOLÓGICAS A região estudada compreende a faixa sedimentar entre Olinda e Goiana, onde ocorre uma sequeencia de rochas cretácicas representada por uma formação basal arenítica (Formação Beberibe) e uma unidade superior calcária (Formação Gramame) (Fig. 2); esta última, estendendo-se segundo uma faixa paralela à costa, com largura máxima de $5 \mathrm{~km}$. Sobre estes sedimentos ocorrem, em áreas restritas, uma formação calcária terciária (Formação Maria Farinhá) e, em extensivas áreas, uma unidade terciária areno-argilosa (Grupo Barreiras), além de aluviões e sedimentos de praia recentes. 
aAcia costeira de pernambuco - paraiba

CORTE GEOLÓgICO TRANSVERSAL BARRA DE CATUAMA - ITAPIREMA

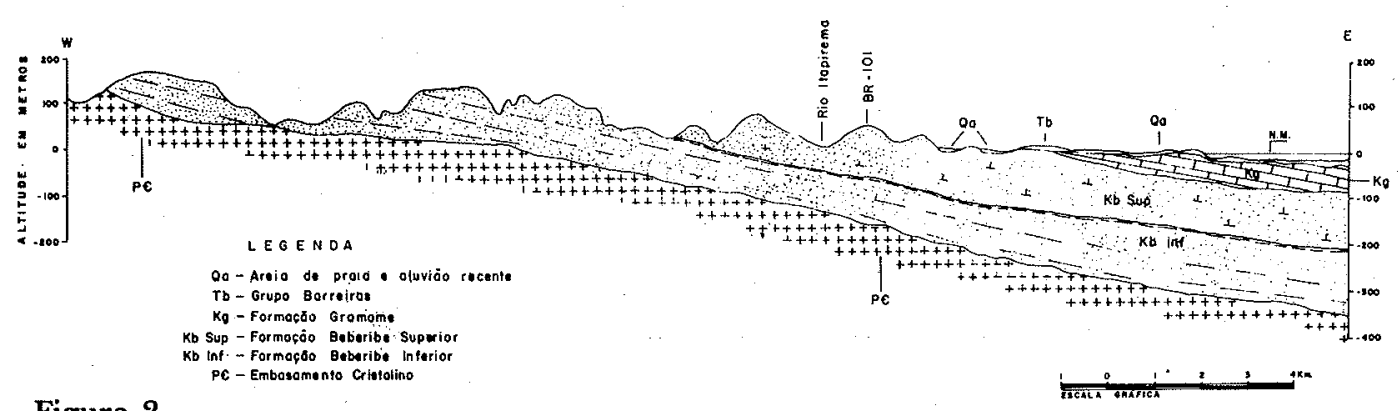

Figura 2

O principal sistema aquífero (Formação Beberibe) é representado na seção inferior por arenitos quartzosos friáveis, de granulaçāo variável, contendo delgadas intercalações sílticas e argilosas; perto da base o arenito tem material silicatado além do quartzo. A seção superior está constituída por arenitos compactos muito calcíferos. O subnível aquífero inferior, classifica-se como um aquífero semiconfinado, limitado na base por um "aquiclude", representado pelo substrato cristalino precambriano e, no topo, por uma camada ou nível semipermeável - folhelhos ou siltitos - que funciona como um "aquitard". Todo o sistema e a superfície do cristalino têm um mergulho regional em direção Leste, e assim a espessura cresce em direção ao mar. A recarga processa-se através de infiltração direta das águas de chuva nas áreas de afloramentos situadas na borda Oeste da bacia e o escoamento geral se verifica em direção à costa. O subnível aquífero superior constitui também um áquífero semiconfinado separado do inferior por um "aquitard" e limitado no topo pelos calcários da Formação Gramame ou pelos sedimentos do Grupo Barreiras. O conjunto é notavelmente anisotrópico, com uma permeabilidade vertical bem menor que a horizontal.

PROGRAMA DE AMOSTRAGEM As amostras para análise de trítio e carbono-14 foram coletadas em poços ou piezômetros por meio de bombeamentos ou vazões livres (poços artesianos surgentes). As águas procediam de diferentes profundidades, tanto do nível inferior como superior do aquífero. Os poços amostrados estão indicados na Fig. 1.

As amostras para análise de carbono-14 foram devidamente processadas no campo, para precipitação de $\mathrm{CO}_{2}, \mathrm{CO}_{3} \mathrm{H}^{-}$e $\mathrm{CO}_{3}^{--} \mathrm{em}$ forma de carbonato, por meio de solução de hidróxido de bário.

As análises de trítio foram efetuadas pelo Instituto de Energia Atômica de São Paulo, enquanto as de carbono-14 foram realizadas pelo Gabinete de Aplicações Nucleares das Obras Públicas de Madrid, através da colaboração do Dr. Antonio Plata.

Infelizmente não há coincidência entre os pontos analisados para carbono-14 e trítio porque durante a análise no IEA ocorreu um acidente no processo de enriquecimento eletrolítico, tendo sido perdidas algumas amostras. Também algumas amostras de carbono-14, obtidas de águas salgadas foram perdidas porque o excesso de $\mathrm{Ba}$ empregado não bastou para precipitar todo o $\mathrm{SO}_{4}^{-}$, deixando grande parte de $\mathrm{CO}_{3} \mathrm{H}^{-}$sem precipitar. 
CONTEÜDO EM TRÍTIO DAS ĀGUAS No Quadro 1 estão indicados os resultados das análises de trítio em 9 amostras de água do Aquífero Beberibe.

Quadro 1 - Conteúdo em trítio das águas subterrâneas do Aquífero Beberibe

\begin{tabular}{lclc}
\hline Poço ou sondagem & $\begin{array}{c}\text { Profundidade } \\
\text { de amostragem } \\
(\mathrm{m})\end{array}$ & \multicolumn{1}{c}{ Zona } & UT (1) \\
\hline PZ-03-IA & 180 & Costeira-Norte & 0,14 \\
PZ-03-IA & 323 & Costeira-Norte & $\sim 0$ \\
PC-02-IA & $100-170$ & Central-Norte & 0,67 \\
PZ-02-IA & $50-60$ & Central-Norte & 0 \\
SN-03-IA & $56-110$ & Central-Norte & 0 \\
IT-03 & $330-384$ & Costeira-Central & 0,41 \\
IG-09 & $84-200$ & Central-Centro & 0 \\
IG-14 & $68-103$ & Central-Centro & 2,78 \\
PL-52 & - & Centro-Sul & 0 \\
\hline
\end{tabular}

(1) $1 \mathrm{UT}=10^{-18} \mathrm{~T} / \mathrm{H}$. As cifras inferiores a $1 \mathrm{UT}$ não são significativamente diferentes de zero

Exceto no poço IG-14, nenhum dos valores obtidos é significativamente diferente de zero, o que indica águas de mais de 20 (vinte) anos de idade, limite de datação do método (Plata, 1972). No poço IG-14, o conteúdo é acentuado, dadas as características de baixa precipitação de trítio na zona, ainda nas épocas de maior aporte atmosférico. Cabé pensar em águas antigas, com uma certa proporção significativa de águas recentes (10 a $30 \%$, talvez). $\widehat{E}$ um poço que penetra $103 \mathrm{~m}$, construído na parte superior do Aquífero Beberibe $(\mathrm{Kb})$, próximo ao contato com a Formação Gramame $(\mathrm{Kg})$. A presença de trítio não é estranha e procede da entrada de águas pouco profundas, e as determinações químicas não se opõem. As características de construção do poço são mostradas na Fig. 3. O poço era inicialmente surgente $\left(30 \mathrm{~m}^{3} / \mathrm{h}\right)$ e atualmente tem o nível estático à cota $2 \mathrm{~m}$. A vazão de bombeamento é de cerca de $150 \mathrm{~m}^{3} / \mathrm{h}$, com 10 a $15 \mathrm{~m}$ de rebaixamento.

As águas com trítio penetram, possivelmente, através da zona telada superior e isto indicaria que a renovação na parte superión do aquífero é rápida.

Conteúdo em radiocarbono das águas No Quadro 2 , estão relacionados os resultados obtidos. A idade indicada pelo lạboratório é a idade aparente, isto é, supondo que o standard NBS de 1950 proporciona 15,3 desintegrações por segundo, por grama, e sem qualquer correção por diluição de carbono não-biogênico. Só foi considerada a eficiência da recontagem, que é de 0,94 . O benzeno sintetizado não foi deixado em repouso o tempo suficiente, dada a urgência das determinações sendo, portanto, esperado que as atividades medidas estejam ligeiramente incrementadas por causa das excitações residuais. 
CARACTERÍSTICAS DE CONSTRUÇÃO DO POCYO IG-14.

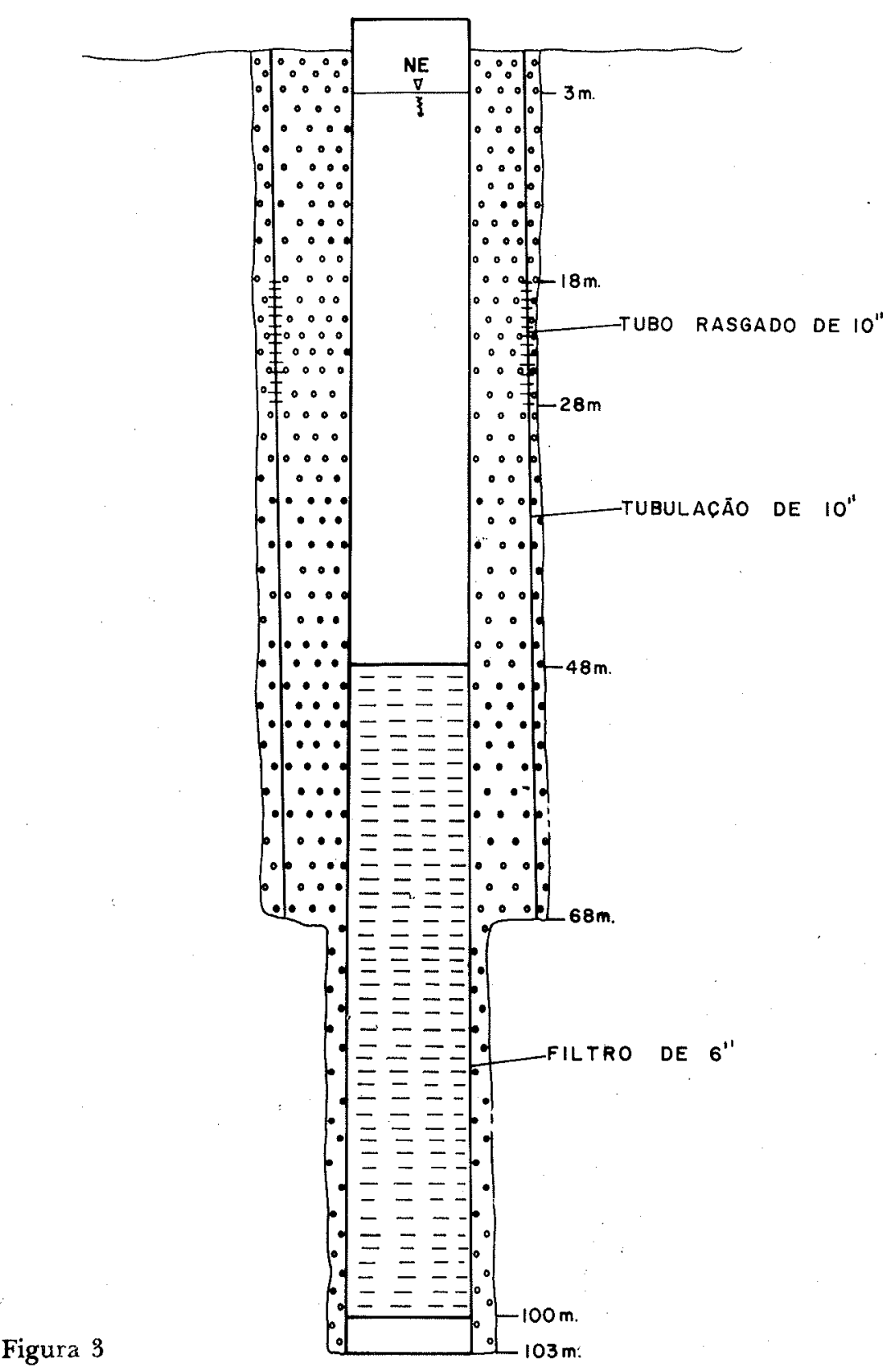


Quadro 2 - Resultados e cálculos relativos ao conteúdo em radịocarbono nas águas subterrâneas do Aquífero Beberibe

\begin{tabular}{|c|c|c|c|c|c|c|c|c|c|c|c|c|c|c|c|c|}
\hline Poşo & Aquifero & Zona & $\begin{array}{r}\text { Idade } \\
\text { Aparen } \\
\text { (anos) }\end{array}$ & & dpsig & $\begin{array}{c}\mathrm{CO}_{2} \\
(\mathrm{ppm})\end{array}$ & $\begin{array}{l}\mathrm{CO}_{3} \mathrm{H} \\
(\mathrm{ppm})\end{array}$ & $\begin{array}{c}\mathrm{Ca} \\
(\mathrm{ppm})\end{array}$ & $\begin{array}{c}\text { Carbono } \\
\text { Tocal } \\
\text { (mmol) }\end{array}$ & $\begin{array}{c}\text { Carbono } \\
\text { bicarbonatos } \\
\text { (mmol) }\end{array}$ & $\begin{array}{l}\text { Fator de } \\
\text { Calculado }\end{array}$ & $\begin{array}{l}\text { orreçāo } \\
\text { Adotado }\end{array}$ & $n_{0}^{d p:}$ & $\mathrm{g}_{\mathrm{n}}$ & $n \cdot n_{0}$ & $\begin{array}{l}\text { Idade } \\
\text { (anos) }\end{array}$ \\
\hline $\begin{array}{l}\text { PC-02-IA } \\
\text { PZ-02-IA }\end{array}$ & $\begin{array}{l}\text { Kb inf } \\
(100-170 \mathrm{~m}) \\
\text { Kb sup }\end{array}$ & Norte, intermediária & $2.150 \pm$ & 500 & 11.05 & 47.1 & $\begin{array}{c}47.6 \\
153,7+\end{array}$ & 2,4 & 1.85 & 0,78 & 0,80 & 0,90 & 13,08 & 11,05 & 0.850 & 1.350 \\
\hline PZ.03-1A & $\begin{array}{l}(50-60 \mathrm{~m}) \\
\text { Kb inf } \\
(323 \mathrm{~m})\end{array}$ & $\begin{array}{l}\text { Norte, intermediária } \\
\text { Norte, costa }\end{array}$ & $\begin{array}{r}5.190 \\
22.000\end{array}$ & $\begin{array}{r}590 \\
1.550\end{array}$ & $\begin{array}{l}7.57 \\
0.94\end{array}$ & $\begin{array}{l}52,8 \\
41,4\end{array}$ & $\begin{array}{l}\left(12 \mathrm{CO}_{3}\right) \\
185,4+ \\
\left(24 \mathrm{CO}_{3}\right)\end{array}$ & $\begin{array}{l}31,2 \\
38,4\end{array}$ & $\begin{array}{l}3,91 \\
4,38\end{array}$ & $\begin{array}{l}2,71 \\
3,44\end{array}$ & $\begin{array}{l}0,65 \\
0.61\end{array}$ & $\begin{array}{l}0.70 \\
0.70\end{array}$ & $\begin{array}{l}10,17 \\
10,17\end{array}$ & $\begin{array}{l}7,57 \\
0,94\end{array}$ & $\begin{array}{l}0,740 \\
0,092\end{array}$ & $\begin{array}{r}2.500 \\
20.000\end{array}$ \\
\hline PZ-03-IA & $\begin{array}{l}\text { Kb sup } \\
(160-180 \mathrm{~m})\end{array}$ & Norte, costa & $>8.80$ & & $<4,81$ & 2.6 & $\begin{array}{c}175,7+ \\
\left(24 \mathrm{CO}_{3}\right)\end{array}$ & 35,6 & 3.34 & 3.28 & 0.54 & 0,65 & 9.45 & 4.81 & 0.510 & 5.600 \\
\hline SN.02-IA & $\mathrm{Kb}$ inf & Sul, recarga & $2.020 \pm$ & 380 & 11,23 & 88.0 & 41.5 & 3,2 & 2,68 & 0.68 & 0.87 & 0.95 & 13.81 & 11.23 & 0.810 & 1.750 \\
\hline PL-02 & Kb sup & Sul, costa & $14.000 \pm$ & 950 & 2.53 & 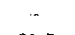 & 239,1 & 49,6 & $4.5(?)$ & 3.92 & $0.55(?)$ & 0.65 & 9.45 & 2.53 & 0.270 & 11.000 \\
\hline OL-68 & Kb inf & Sul, costa & $16.600 \pm$ & 1.300 & 1,83 & 31,7 & & interm. & 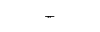 & -. & - & 0,70 & 10,17 & 1.83 & 0.180 & 14.000 \\
\hline OL-14 & Kb sup & Sul, costa & $11.300 \pm$ & 1.450 & 3.54 & 16.7 & 214,7 & 92,8 & 3,90 & 3.52 & 0,55 & $0,60^{\circ}$ & 8,72 & 3.54 & 0,410 & 7.400 \\
\hline
\end{tabular}

OBS.: - As amostras foram coletadas entre 15/05/75 e 29/09/75, precipitando o carbono solúvel em forma de $\mathrm{CO}_{2}+\mathrm{CO}_{3} \mathrm{H}^{-}+\mathrm{CO}_{3}=$, em forma de $\mathrm{CO}_{3} \mathrm{Ba}$, utilizando soluşāo de $\mathrm{Ba}$;

- dps = desintegracōes por segundo/grama;

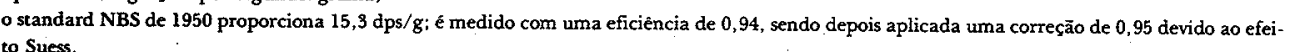


A correção por carbono não-biogenico (carbono dos calcários c carbonatos do terreno, supostamente iscnto do $(-1 \cdot 1)$, pode ser realizada segundo a seguinte expressão:

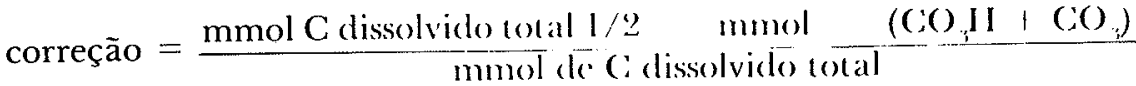

Não obstante, essa correção não ć suficionte, já que os arenitos do aquífero da área de recarga e do aquífero profundo estão isentos de carbonatos, como é indicado pelo baixo conteúdo em Ca na água. A transformação de $\mathrm{CO}$, em $\mathrm{CO}, \mathrm{H}$ foi realizada, em parte, por alteração de silicatos, o que não permite a incorporação de carbono não-biogênico. Por esse motivo, no Quadro 2, foi modificado o fator de correção para considerar tal efeito, além dos possíveis câmbios isotópicos. $\mathrm{O}$ fator de cor. reção adaptado é utilizado para o cálculo da idade provável, segundo:

idade $=8300 \ln \frac{\mathrm{n}_{0}}{\mathrm{n}}, \quad$ (em anos)

onde:

$\mathrm{n}_{\mathrm{o}}=$ atividade do standard $=15,3 \times 0,95 \times 0,94 \times 0,94 \times$ fator corre ção, sendo:

$0,95=$ correção por efeitos Suess (diluição do carbono atmosférico por carbono não-biogênico de queima de combustíveis fósseis, em $1950)$;

$0,94=$ eficiência de recontagem;

$\mathrm{n}=$ atividade medida na amostra.

Não foram realizadas determinações de C-13 para correção dós valores de C-14.

No Quadro 3, estão comparados esses resultados com o conteúdo em trítio e em cloretos. A interpretação correspondente apresenta certas dificuldades, devido à escassez de dados e a não-coincidência dos pontos com análises de trítio com os que possuem C-14.

Quadro 3 - Conteúdo em radiocarbono nas águas subterrậneas do Aquífero Beberibe

\begin{tabular}{|c|c|c|c|c|c|}
\hline Poço & Aquífero & Zona & $\begin{array}{c}\text { ppm de } \\
\mathrm{Cl}\end{array}$ & Anos & Trítio \\
\hline $\begin{array}{l}\text { PC-02-IA } \\
\text { PZ-02-IA } \\
\text { PZ-03-IA } \\
\text { PZ-03-IA }\end{array}$ & $\begin{array}{l}\mathrm{Kb} \inf (100-170 \mathrm{~m}) \\
\mathrm{Kb} \sup (50-60 \mathrm{~m}) \\
\mathrm{Kb} \inf (323 \mathrm{~m}) \\
\mathrm{Kb} \sup (160-180 \mathrm{~m})\end{array}$ & $\begin{array}{l}\text { Norte, intermediária } \\
\text { Norte, intermediária } \\
\text { Norte, costa } \\
\text { Norte, costa }\end{array}$ & $\begin{array}{l}17 \\
22 \\
27 \\
17\end{array}$ & $\begin{array}{r}1.350 \\
2.500 \\
20.000 \\
>5.600\end{array}$ & $\begin{array}{l}0,67 \\
0 \\
0,14 \\
\sim 0\end{array}$ \\
\hline $\begin{array}{l}\text { SN-02-IA } \\
\text { PL-02 } \\
\text { OL-68 } \\
\text { OL-14 }\end{array}$ & $\begin{array}{l}\text { Kb inf } \\
K b \text { sup } \\
K b \text { inf } \\
\text { Kb sup }\end{array}$ & $\begin{array}{l}\text { Sul, recarga } \\
\text { Sul, costa } \\
\text { Sul, costa } \\
\text { Sul, costa }\end{array}$ & $\begin{array}{c}15 \\
55 \\
- \\
304\end{array}$ & $\begin{array}{l}1.750 \\
1.100 \\
1.400 \\
7.400\end{array}$ & $\begin{array}{l}- \\
- \\
- \\
-\end{array}$ \\
\hline
\end{tabular}


$\mathrm{Na}$ área norte, a ausência de trítio se corresponde bem com aș elevadas idades da água. Ao passar da faixa intermediária para a costeira, as idades crescem rapidamente. Isso assinala que, na costa, a água profunda é praticamente estacionária; não é possível deduzir a velocidade do movimento vertical, posto que um dos dados é incerto, face a seu escasso conteúdo em carbono. De qualquer modo, a permeabilidade vertical parece ser menor que $10^{-4} \mathrm{~m} /$ dia.

$\mathrm{Na}$ faixa intermediária, a idade é ainda elevada, devido a que a profundidade dos pontos amostrados afeta o fluxo regional e não o fluxo local, que descarrega nos pequenos rios e mananciais. Essa elevada idade parece justificar supor a existência, ali também, de um fluxo vertical ascendente, o qual, em primeira aproximação, percorreria $80 \mathrm{~m}$ em cerca de 1000 anos, com um gradiente vertical natural (que não coincide com o atual) da ordem de $5 \mathrm{~m} \mathrm{em} 80 \mathrm{~m}$. Para uma porosidade de 0,10 , a permeabilidade vertical seria:

$$
\frac{80 \mathrm{~m}}{1000 \times 365 \text { dias }} \times 0,10=\mathrm{K} \frac{5 \mathrm{~m}}{80 \mathrm{~m}} \rightarrow \mathrm{K}=3,5 \times 10^{-4} \mathrm{~m} / \text { dia }
$$

Na zona sul, são verificados os mesmos fenômenos, com águas muito antigas na zona costeira. Na zona de recarga, a água amostrada tem cerca de 1750 anos, o que indica, tal como era de esperar, que a circulação se reduz à parte superior do aquífero e que, nos níveis profundos, o movimento é muito lento, tanto mais lento quanto mais próximo da costa, onde a água é quase estacionária. O poço OL-14 mostra um elevado conteúdo em cloretos e, em menor grau, também o PL-02. Em ambos, as idades, segundo o C-14, são elevadas; porém, não se dispõe de uma análise correlativa de trítio, o que não possibilita elucidar se a salinização é proveniente de um movimento horizontal de águas do aquífero profundo, ou de uma pequena entrada superior, por deficiências de construção do poço, ou de caminhos verticais preferenciais no aquífero. Seria muito recomendável uma nova amostragem de detalhe nessa zona. Sem dúvida, e com caráter provisório, parece mais verossímil a primeira hipótese, ou seja, a de movimento lateral horizontal.

A questão de que o poço com água mais salina (OL-14, $304 \mathrm{ppm} \mathrm{de} \mathrm{Cl}$ ) tenha uma idade pouco menor que a dos outros poços costeiros, além de sua menor profundidade, admite três explicações, baseando-se em que essa é a zona de maior exploração:

a) Trata-se de água que circula, em sua maior parte, desde a área de recarga, com uma porção menor de águas antigas do lado costeiro e sob o mar, que são as que proporcionam um aumento de salinidade. A explotação de $Q=10^{6} \mathrm{~m}^{3} /$ ano localizado, durante $t=10$ anos e utilizando, de preferência, um setor de $90^{\circ}$, em uma espessura de $b=100 \mathrm{~m}$ e porosidade $\mathrm{m}=0,1$, produz um deslocamento $\mathrm{r}$ de

$\mathrm{Qt}=\frac{\pi \mathrm{r}^{2} \mathrm{bm}}{4}$

$r=\frac{10^{6} \times 10 \times 4}{\pi \times 100 \times 0,1}=1130 \mathrm{~m}$

que não é suficiente para produzir alterações substanciais na idade. Desse modo, o mécanismo parece pouco provável. 
b) Existe a entrada de águas salgadas superficiais. Para produzir uma notável diminuiçāo de idade, com somente um aporte moderado de cloretos, deve se tratar de águas salobras ou salgadas antigas, sobre as quais não se têm informações, já que são desconhecidas as características das águas subterrâneas pouco profundas da zona. De todo modo, uma diminuição da idade desde 14000 a 7400 anos, isto é, desde $1,83 \mathrm{dps} / \mathrm{g}$ a $3,54 \mathrm{dps} / \mathrm{g}$, supõe uma contribuição de

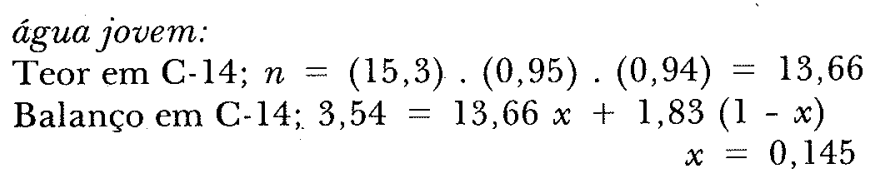

ou seja, 14,5\% é de água jovem.

água de 2500 anos:

Teor em C-14; $n=7,6$

Balanço em C- $14 ; 3,54=7,6 x+1,83(1-x)$

$x=0,30$

ou seja, $30 \%$ é de água de 2500 anos.

Supondo uma água inicial de 20 ppm de Cl e uma água final de 304 ppm de Cl, a água infiltrada deveria ter

1. Caso:

Balanço de $\mathrm{Cl} ; 304=20(1-0,145)+0,145 \mathrm{C}$

$\mathrm{C}=1980$ ppm de $\mathrm{Cl}$

2. Caso:

Balanço de $\mathrm{Cl} ; 304=20(1-0,30)+0,30 \mathrm{C}$

$\mathrm{C}=967$ ppm de $\mathrm{Cl}$

Verifica-se que não parece provável que se trate de uma infiltração direta de água marinha, senão, em todo caso, de água salobra do aquífero, da qual não se tem dados diretos sobre sua existência, embora medidas de resistividade por meio de - sondagens elétricas tenham indicado a presença de águas salinas áté a profundidade de 25-30 metros.

c) A idade é menor, visto que o poço OL-14 está situado muito ao sul, onde a bacia sedimentar se estreita marcadamente e é provável que a circulação seja maior, por ser a permeabilidade vertical maior (Custódio, 1976).

A solução mais verossimel é, provavelmente, uma combinação dos mecanismos indicados nas alíneas (b) e (c), embora existam muitas dúvidas.

CONCLUSÕES O conteúdo em trítio das águas subterrâneas não é significativamente diferente de zero, na maior parte das amostras, o que indica águas de mais de 20 anos de idade.

- O conteúdo em radiocarbono indica águas muito antigas (até 20.000 anos), especialmente nos níveis inferiores da faixa costeira, o que evidencia que a água profunda é praticamente estacionária à escala de tempo humana.

- Na faixa de transferência ou intermediária, a idade é ainda elevada, o que parece indicar a existência, também nestas áreas, de um fluxo vertical ascendente o 
qual, em primeira aproximação, percorreria 80 metros em cerca de 1000 anos com um gradiente vertical natural da ordem de 5 metros em 80 metros. Para uma porosidade de 0,10 a permeabilidade vertical seria $\mathrm{K}=3,5 \cdot 10^{-4} \mathrm{~m}$.

- Na zona de recarga, a água amostrada tem cerca de 1750 anos (zona sul), o que indica que a circulação se reduz à parte superior do aquífero (conforme se verifica pela contribuição aos rios) e que nos níveis profundos o movimento é muito mais lento.

- Não há evidências de contaminação de águas marinhas por infiltração direta, podendo ocorrer, todavia, pequena entrada de águas salobras mais superficiais, embora não se tenha dados diretos sobre sua existência.

\section{BIBLIOGRAFIA}

PLATA, A. - 1972 - Isotopos en Hidrologia. Editorial Alhambra, Colección Zairos, Bilbao, 328 p.

CUSTODIO, E. - 1976 - Relaciones Agua Dulce-Agua Salada en las Regiones Costeras. Hidrologia Subterranea, Ed. E. Custodio e M.R. Llamas, Ediciones Omega, Barcelona, Sección 13, 2 : 1313-1389.

CUSTODIO, E. - 1976 - Datacion y Trazado Natural y Accidental de las Aguas Subterraneas. Hidrologia Subterranea, Ed. E. Custodio e M.R. Llamas, Ediciones Omega, Barceloná, Vol. II, cap. 12.4, pp., 1245-1287. 